\title{
Two Stages Scheduling of Steel Making using Earliest Deadline First Algorithm
}

\author{
P.Chandra Sekhar ${ }^{\# 1}$, PS Avadhani ${ }^{\# 2,}$ P. Suresh Varma ${ }^{\# 3}$ and NRMRBhargava ${ }^{\# 4}$ \\ ${ }^{1}$ Dy. General Manager, IT Department, Visakhapatnam Steel Plant, RINL,A.P, India, \\ ${ }^{3}$ Department of CSE, Adikavi Nannaya University, A.P, India \\ cspulle@vizagsteel.com \\ 3.vermaps@yahoo.com \\ ${ }^{2}$ Department of CS,Andhra Univrsity, Vizag, A.P,India, ${ }^{4}$ Department of Metallurgy, Andhra University, A.P, \\ 2psavadhani@yahoo.com, \\ ${ }^{4}$ nrmrbhargava@rediffmail.com
}

Abstract: Steel making is a process of converting hot metal into liquid steel by removing impurities by oxidation process. In this process Oxygen is blown into hot metal to oxidize impurities like carbon, Manganese, Sulhpur, Phosphorous and it will form into a slag. After tapping the steel into the ladle Ferro Alloys are added to achieve the target grade of the steel. The liquid steel is sent to Argon Rinsing station for homogenizing the steel and killing the steel to remove dissolved oxygen. In the continuous casting process the homogenized steel is allowed to flow into tundish and from there into bottom less mould. The semi cooled piece of bloom is continuously drawn out of mould and cut into required length pieces. Under one tundish several moulds may be available as per design.

Scheduling a Steel making process is a very complicated and it involves lot of equipment and dynamism in nature. Lot of research is ongoing to schedule the steel making process and many attempts have been made using different kinds of algorithms.

In this paper the authors have attempted to schedule steel making process at LD and ARS Processes. Different grades are produced in LD Converter based on the Hot Metal supply. Hot metal supply comes from the blast furnace in the form of Jobs. These jobs allocated to the LD Converters using Earliest Dead line first (EDF) scheduling algorithm. The output of $L D$ converters are further processed in Argon Rinsing Stations(ARS) and send to continuous casting machines (CCM) to cast into Blooms. The Heats/Jobs arrived ARS are scheduled based on EDF Scheduling. The EDF algorithm used at LD and ARS have shown better performance metrics when compared with other conventional models metrics.

In this paper, the authors have designed and developed a scheduling model based on Earliest Dead Line First. The authors have evaluated performance metrics of the model like Turnaround time, Average waiting time and dead line deviation.

These results indicated that the scheduling model has shown a significant improvement of EDF on the job scheduling when compared with conventional methods like First Cum First scheduling (FCFS), Shortest Job First (SJF).

\section{INTRODUCTION}

Steel making is a process of converting Hot Metal into liquid steel by blowing oxygen in a converter having a capacity about $150 \mathrm{~T}$. The impurities will get oxidized and called as slag floating on the liquid steel having temperature $1700 \mathrm{C}$. The alloying additions are added into ladle after tapping depending on the grade of steel to be produced. The blowing time depends on the silicon in the hot metal and grade to be produced. Steel is tapped into ladles from converter and sent to Argon Rinsing Station (ARS). In the Argon Rinsing Station (ARS) the steel is rinsed with Argon to homogenize the steel and drive out dissolved oxygen. Killing agents are added to deoxidize the steel. After rinsing the steel is sent to Continuous casting machines for casting.

In the continuous casting the liquid steel in the ladle is kept on turret stand and poured into tundish which is having nozzles at the bottom. These nozzles are opened to bottom-less moulds and the partially the continuous casting solidified metal is drawn continuously from the bottom and cut to length at the gas cutting machine. Different grades of steel require different super heat based on the TTT curve and accordingly time of casting varies with the grade being cast. The speed of casting depends on the super heat and grade.

The total steel making process involves the following important steps.

1. Blowing of Hot metal in LD converter about 150 tons of steel is produced

2. In the ARS Ferro Alloys are added to adjust the alloying elements to reach the aim Grade of the steel

3. Rinsing of the Steel with Argon to homogenize the steel and to kill the steel to remove the oxygen content. Sometimes temperature of the steel is raised to meet the requirement of casting. Generally 
about $50^{\circ} \mathrm{C}$ super heat is maintained in the steel above liquidous temperature based on the carbon equivalent

4. In the Continuous Casting Machine Liquid steel is cast into Blooms/Slabs/Billets by pouring steel into bottom less moulds and drawing the semi solidified blooms out and cut into required length in a gas cutting machine.

The scheduling of steelmaking continuous-casting (SCC) processes is of major importance in iron and steel operations, since it is often a bottleneck in iron and steel production. Optimal scheduling of SCC processes can increase profit, minimize production cost, reduce material and energy consumption, and improve customer satisfaction. All these parameters performance metrics mostly depends on the type of scheduling of heats in LD Converters and ARS stations Scheduling of SCC involves sequencing of jobs on LD Converter, Argon Rinsing and Continuous Casting in a non-preemptive so that total elapsed time and average waiting time are minimum. The scheduling of Steel Making and Continuous Casting involves sequencing of heats based on the grade to be made and timings in each unit to be spent which depends on the service rate at LD Converters and ARS.

The steel that is produced in one treatment time is called a Heat. The number of heats of different steel grades is produced in steel melting shop in a day and the different heats to be produced is known wellin advance. In the Steel Melting shop, these heats are being planned randomly without any logic and more or lesson first cum first basis. The heats one by one gets processed in LD Converter and sent to argon rinsing station for further processing. In this research, authors have focused on mainly different schedulingalgorithms of the heats so that effective utilization of the machines increased and waiting time is reduced.

As The authors tried to explore other different models for the heats scheduling in steel making to improve the productivity, effective utilization of machines and cost of production. The authors have tried Shortest Job First (SJF), Earliest Deadline First (EDF) for the two machines i.e LD converters and Argon Rinsing Stations(ARS) and compared with FCFS and SJF scheduling. The metrics like Turnaround time, Average waiting time and dead line deviation which are used to evaluate performance of the machines are comparedfor the different models for effectiveness of each model over the other. For data validation and verification number of heats/Jobs data taken from the Steel Plant Production data.

These results indicated that the EDF scheduling model has shown a significant improvement of over First Come First Scheduling (FCFS), Short Test Job First (SJF). This indicated that in the steel making the model EDF has given effective utilization of LD Converters and Argon rinsing stations and improvement of productivity over presently being used FCFS modeling.

\section{RELATED WORK}

Liro Harjunkoski, Guido Sand (2000) have presented MILP-models for melt shop scheduling optimization that can be flexibly adapted to different plant structures. Moreover, the flexibility allows for modeling individual characteristics of parallel equipment, particular processing and changeover times, scarce resources and maintenance requests. Vikas Kumar and etal (2000),formulated the scheduling problem in steel making as a linear integer program to determine the scheduling sequence for differentcharges. Bids are then obtained for sequencing the charges and a heuristicapproach is used to evaluate the bids. The proposed algorithm has beenverified by a case study.

X.F.Li etal (2001), presented a new modeling method that is called Extended Timed Synchronized Colored PN (ETSCPN) to set up the dynamic model of the whole system. Further a hierarchic algorithm was presented to solve the dynamic scheduling of the model. The simulation result demonstrated that the algorithm is efficient and fit for the dynamic scheduling of a class of multiple stages and multiple machines and FIFO system. Dario Pacciarelli etal(2004), formulated production planning based on the alternative graph which details the constraints relevant for the scheduling problem. The problem of scheduling is solved by beam search procedure and compared the results with a lower bound of the optimal solutions and with actual performance obtained in the plant.

PA Guegler and FJ Vasko et al(2007) presentedan efficient domain-specific heuristic is combined with metaheuristic approaches in a prototype scheduling model. Specifically, given preliminary schedules for the continuous casters, the model determines the allocation, sequencing, and scheduling of batches of steel at the basic oxygen steelmaking furnaces, the degassing facilities, and the ladle treatment stations. It also makes the appropriate schedule modifications at the continuous casters. Computational results are presented.Kebin Lu etal (2008), presented a hybrid heuristic and optimization algorithms for the integrated scheduling problem of steelmaking and continuous casting. The scheduling system had been embedded into the whole MES of a big iron and steel group in China. They have achieved the satisfactory results.

Hubert Missbauer etal (2009), presented the models, algorithms and implementation results of a computerized scheduling system for the steelmaking-continuous casting process of a steel plant in Austria. Extensive numerical tests with real-life data and more than two years of experience with the implementation 
demonstrate that the system produces reasonable schedules and is accepted by the planners. Arezoo Atighehchian et al. (2000), presented steel-making continuous casting (SCC) scheduling problem (SCCSP). They have developed algorithm, named HANO, is based on a combination of ant colony optimization (ACO) and non-linear optimization methods. The efficiency of HANO is compared with heuristic and genetic algorithms as a real case used at Mobarakeh Steel Company (MSC), the biggest steel factory in the Middle East. Numerical results reveal the higher efficiency of the developed approach compared with the heuristic one used at MSC.

H. B. Wang and etal (2010) presented a genetic algorithm model for production scheduling as JSSP with fuzzy processing and delivery time.The test results show that the improved genetic algorithm can find better solution. Xiu-ying wang etal (2010), presented a model for scheduling steel-making and continuous casting combining the mathematical programming, fuzzy logic, expert system based techniques. The results demonstrated that the proposed scheduling strategy to some extent satisfy the requirement of practical production.

IESLi,Xin Xiao etal (2012), developed a novel unit-specific event-based continuous-time mixedinteger linear optimization (MILP) model for this problem and incorporate several realistic operational features. Four large-scale industrial problems are used to illustrate the efficiency and effectiveness of the proposed formulation and rolling horizon approach. The computational results show that the extended rolling horizon approach successfully solves the large-scale case studies and results in the same or better integer solution than that obtained from directly solving the entire scheduling model.

Worapradya.K, Thanakikasem P etal [2014], formulateda new optimization model that more closely represents real-world situations, and a hierarchical genetic algorithm (HGA) tailored particularly for searching for an optimal Steel Making-Continuous Casting Schedule. The optimization model is developed by integrating two main planning phases of traditional scheduling: (1) planning cast sequence, and (2) scheduling of steelmaking and timing of all jobs. A Worapradya, Purit Thanakijkasem etal. [2015], presented an artificial neural network (ANN) based model adopted with a challenge of designing an accurate model due to the model complexity from the discrete and nonlinear properties of the system performance. The experimental result shows that the proposed methodology is successful in designing a robust schedule that provides a lower production cost under an acceptable breakdown probability.

In the literature, very little work on Scheduling of Steel Making and Continuous Casting (SCC) reported. In the available literature researchers have focused on Artificial Intelligence and Nueral networks to solve the SCC scheduling problem. No author reported the research on SCC Scheduling using Deadline Concept. Deadline aware Scheduling concept is most important concept being used in all industrial scheduling activities. Hence authors have chosen EDF methodology for scheduling of heats to LD converters and ARS stations of steel making.

\section{EARLIEST DEADLINE FIRST ALGORITHM}

Steel production is an extremely complex process and determining coherentSchedules for the wide variety of production steps in a dynamic environment,where disturbances frequently occur, is a challenging task. In the steel productionprocess, the blast furnace continuously produces liquid iron, which is transformedinto liquid steel in the melt shop. The majority of the molten steel passes througha continuous caster to form large steel blooms, which are rolled into coils/angles/bars/rounds/structural in Wire rod Mill, LMMM, MMSM mills. The scheduling system of these processes has very different objectivesand constraints, and operates in an environment where there is a substantialquantity of real-time information concerning production failures and customerrequests. The steel making process, which includes steel making followedby continuous casting, is generally the main bottleneck in steel production. Therefore, comprehensive scheduling of this process is critical to improve thequality and productivity of the entire production system.In this research authors considered the output of Blast Furnace i.e Hot Metal as input to LD Converter and the output of LD Converters is the output to ARS Stations.

The scheduling of Steel Making and Continuous Casting involves sequencing of heats based on the grade to be made and timings in each unit to be spent. Deriving an optimized sequencing of the Heats/Jobs from converter to Caster will give more productivity and optimum machine utilization. The aim of the research is to derive an optimized scheduling based on the timings of blow, rinsing and casting which is depends on the many metallurgical constraints.

In this paper authors have consideredfor two machines of steel making namely LD Converter and Argon Rinsing Unit. In this research it is considered thatthere are ' $\mathrm{m}$ ' number of converters parallely operating and processing heats and there are ' $n$ ' number of Argon Rinsing Units parallely processing heats. The model randomly determines a factor for each job/heat and finds out a deadline time for each job/heat. After deadlines are arrived for each heat / job the jobs are ordered based on the deadline function in ascending order is 
sequenced. The scheduling model considers for each 4 jobs/heats it sequences on one stream of LD converter (M1) and Argon rinsing station (M2). The concept of multiple machine scheduling is shown in Figure 1.

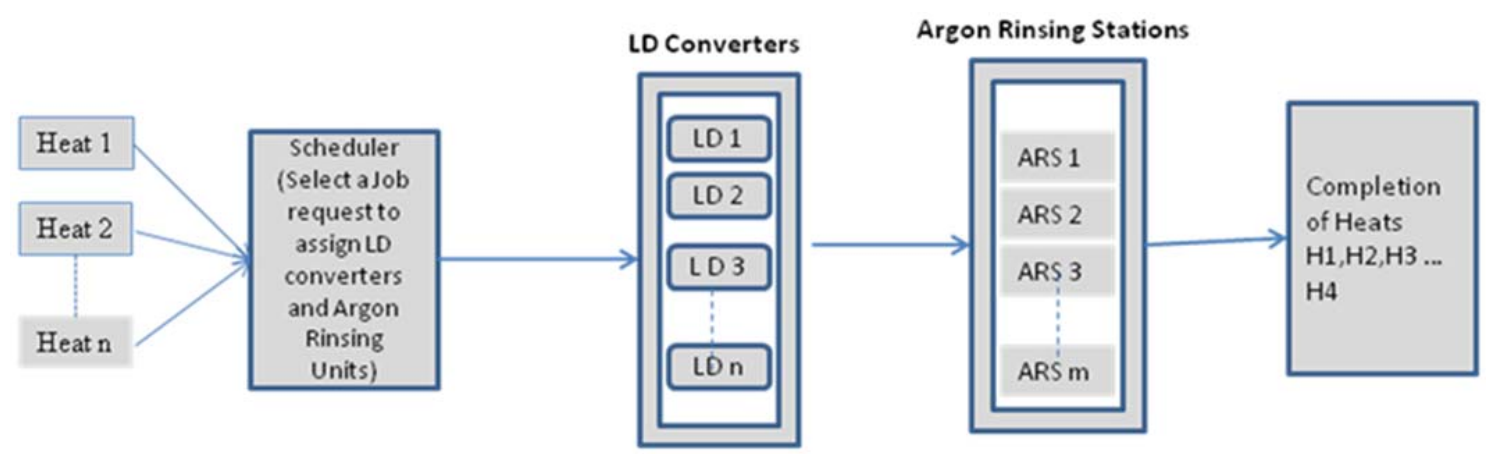

Fig. 1 Depiction of scheme of scheduling activity in LD Converters and Argon Rinsing Stations in a Steel Melting Shop

Deadline time for each job/heat is calculated based on the total time it takes to process each heat in the LD converter(M1) and Argon Rinsing Station (M2) and multiplied with random number $\alpha$. The deadline function is defined as $\beta(\mathrm{M} 1+\mathrm{M} 2)+\gamma *$ deadline time where $\beta, \gamma$ are factors decided based on the best optimization and $\beta+\gamma=1$. The schedule sequence is arranged in ascending order as per deadline function value. The ordered sequence is split into 12 sets of sequences and each set shall have 4 jobs/heats. In each set start time, waiting time and deadline variation is computed. If the dead line variation is minimum, that scheduling sequence is declared as best optimized sequence.

\section{METHODOLOGY}

In this paper the authorstook 48 jobs with Processing Times on $\mathrm{M}_{1}$ and $\mathrm{M}_{2}$ i.e LD Converter Blowing time $\left(t_{1}\right)$ and Argon Rinsing time $\left(t_{2}\right)$ as shown in table 4.1.The deadline time for each job is calculated using function $\alpha^{*}\left(t_{1}+t_{2}\right)$ where $t 1,2$ are processing times and $\alpha$ is random number varies between 2 and 4 . The scheduling at LD converter and ARS stations is done using EDF methodology on the data presented in Table 4.1 and it is compared with FCFS and SJF. Various Performance metrics like Average Turnaround Time (ATT), Average Waiting Time (AWT), Total Elapsed Time (TET) and dead line deviation is computed and compared. Java simulation programme is developed to compute all scheduling methods. Out of 48 jobs, it is considered that each 4 jobs/heats will run on one stream of Virtual machines M1 and M2 accordingly the computations are done. So that starting times after four jobs / heats will become zero for the first job/heat in that stream.

Waiting Time of a job request is the time elapsed between the arrival time of job request and whenthe job request starts its work on Machine of type-1, plus the time elapsed between the timeit completes its work on Machine of type-1 and starts its work on Machine of type-2.The Total Elapsed Time of the entire schedule is the time when all job requests completed theirwork on both machines of type- 1 and type- 2 respectively. Total Elapsed time of thisschedule is the $c_{k}$, where $\mathrm{k}$ is the last request in the schedule given by the scheduling algorithm. The performance metrics can be computed by the following computations for a given schedulingsequence.Average Waiting Time (AWT), Average Turn-around Time(ATT), Total Elapsed Time (TET) of alljob requests can be computed as follows.

$$
\begin{array}{r}
A W T=\sum_{i=1}^{i=n}\left(\left(c_{i}-t_{i 2}\right)-\left(s_{i}+t_{i 1}\right)\right) / \mathrm{n} \\
A T T=\left(\sum_{i=1}^{i=n} c_{i}\right) / n
\end{array}
$$

Where $\mathrm{n}:$ Number of jobs.

i : Job Request Number

$t_{i 1} \quad$ : The time required on Machine of type-1 (M1) for job request ii.

$t_{i 2}$ : The time required on Machine of type-2 (M2) for job request ii.

$c_{i} \quad$ : Completion time of job request i Machine of type-2.

$\mathrm{TET}=c_{k}$, where $\mathrm{k}$ is the last job request in schedule.

The authors have considered 48 Jobs on 2 types of Machines by considering 12 instances are available for each machine type. The dead line time is calculated using simulation programme for each heat or job id as $(\mathrm{M} 1+\mathrm{M} 2) *$ Alpha. This is shown in Table I and algorithm used for EDF scheduling is shown in Algorithm 3.1. Where Alpha is the random number between 2 and 4 . The dead line indicates the job to be completed within that time otherwise dead line deviation which is shown in Table 4.2. 
TABLE: I. The Job Id, M1 , M2 , Alpha and Dead Line values

\begin{tabular}{|c|c|c|c|c|}
\hline Job ID & M1 & M2 & Alpha (2 to 4) & Dead Line $($ M1+M2) * Alpha \\
\hline 0 & 15 & 32 & 2 & 94 \\
\hline 1 & 19 & 25 & 4 & 176 \\
\hline 2 & 17 & 25 & 4 & 168 \\
\hline 3 & 16 & 20 & 3 & 108 \\
\hline 4 & 17 & 20 & 2 & 74 \\
\hline 5 & 18 & 27 & 2 & 90 \\
\hline 6 & 18 & 12 & 3 & 90 \\
\hline 7 & 20 & 32 & 3 & 156 \\
\hline 8 & 19 & 17 & 4 & 144 \\
\hline 9 & 18 & 42 & 3 & 180 \\
\hline 10 & 17 & 14 & 2 & 62 \\
\hline 11 & 19 & 10 & 2 & 58 \\
\hline 12 & 15 & 17 & 3 & 96 \\
\hline 13 & 18 & 22 & 4 & 160 \\
\hline 14 & 17 & 18 & 2 & 70 \\
\hline 15 & 16 & 28 & 2 & 88 \\
\hline 16 & 16 & 25 & 3 & 123 \\
\hline 17 & 15 & 12 & 2 & 54 \\
\hline 18 & 17 & 22 & 4 & 156 \\
\hline 19 & 18 & 24 & 3 & 126 \\
\hline 20 & 15 & 14 & 3 & 87 \\
\hline 21 & 18 & 32 & 4 & 200 \\
\hline 22 & 17 & 95 & 2 & 224 \\
\hline 23 & 17 & 19 & 2 & 72 \\
\hline 24 & 23 & 19 & 3 & 126 \\
\hline 25 & 18 & 25 & 3 & 129 \\
\hline 26 & 10 & 19 & 3 & 87 \\
\hline 27 & 17 & 23 & 4 & 160 \\
\hline 28 & 17 & 14 & 3 & 93 \\
\hline 29 & 18 & 22 & 2 & 80 \\
\hline 30 & 22 & 14 & 3 & 108 \\
\hline 31 & 17 & 35 & 2 & 104 \\
\hline 32 & 17 & 22 & 2 & 78 \\
\hline 33 & 27 & 15 & 2 & 84 \\
\hline 34 & 18 & 8 & 3 & 78 \\
\hline 35 & 17 & 10 & 2 & 54 \\
\hline 36 & 18 & 9 & 4 & 108 \\
\hline 37 & 18 & 23 & 4 & 164 \\
\hline 38 & 17 & 11 & 3 & 84 \\
\hline 39 & 20 & 12 & 3 & 96 \\
\hline 40 & 21 & 25 & 4 & 184 \\
\hline 41 & 17 & 14 & 3 & 93 \\
\hline 42 & 18 & 32 & 3 & 150 \\
\hline
\end{tabular}




\begin{tabular}{|c|c|c|c|c|}
\cline { 4 - 5 } 43 & 18 & 10 & 2 & 56 \\
\hline 44 & 18 & 13 & 2 & 62 \\
\hline 45 & 17 & 20 & 4 & 148 \\
\hline 46 & 17 & 20 & 2 & 74 \\
\hline 47 & 10 & 25 & 3 & 105 \\
\hline
\end{tabular}

\section{ALGORITHM I}

The algorithm for generating scheduling sequences and calculation of the above results is shown below.

\begin{tabular}{ll}
\hline Algorithm & $:$ Pseudo-code for EDF Scheduling Algorithm \\
Input & $:$ n number of job requests with processing time's $\mathrm{t}_{\mathrm{i} 1}$ and $\mathrm{t}_{\mathrm{i} 2}$ on \\
& Two types of M1 and M2 Machine \\
& $\mathrm{p}$ number of instances are available for each Machine \\
& $\mathrm{d} 1_{\mathrm{i}}$ is deadline of $\mathrm{i}^{\text {th }}$ job request $=$ Alpha $*\left(\mathrm{t}_{\mathrm{i} 1}+\mathrm{t}_{\mathrm{i} 2}\right)$ \\
& $2 \leq$ Alpha $\leq 4$ \\
& $($ Beta + Gama $)=1$ \\
Output $:$ Optimal Scheduling sub sequences $\mathrm{Seq}_{1}, \mathrm{Seq}_{2}, \mathrm{Seq}_{3, \ldots .} \mathrm{Seq}_{\mathrm{p}}$
\end{tabular}

1. begin

2. $\mathrm{i}=0$;

3. solution vector $=$ empty;

4. for each job request $r_{i}$ with minimum $\left(B e t a * d_{i}+\operatorname{Gama}^{*}\left(\left(t_{i 1}+t_{i 2}\right)\right)\right.$ among all unprocessed jobs do

5. $\quad$ add the job request $r_{i}$ to the solution vector at index $i$;

6. $\quad \mathrm{i}=\mathrm{i}+1$;

7. end for;

8. for $\mathrm{i}=0$ to $\mathrm{n}-1$ do

9. $\mathrm{j}=\mathrm{i} \% \mathrm{p}$;

10. append solution vector[i] to the scheduling sub sequence $\mathrm{Seq}_{\mathrm{j}}$;

11. end for;

12. for $\mathrm{i}=1$ to $\mathrm{p}$ do

13. calculate performance metrics for each scheduling sub sequence $\mathrm{Seq}_{\mathrm{i}}$;

14. end for;

15. calculate aggregate performance metrics for the entire scheduling sequence;

16. end;

The AlgorithmI shows the methodology in computing performance metrics using EDF, FCFS and SJF scheduling and the results are shown in Table I.

\section{ANALYSIS AND COMPARISION OF RESULTS}

Using the Java simulation programming the FCFS, SJF and EDF scheduling results computed and the results are tabulated below.

\section{A. FCFS Scheduling Results:}

Forty eight jobs/Heats of different grades are scheduled on First Cum First Basis and there are scheduled on LD Converters First (M1) and Argon Rinsing Units next (M2). The start time of Job/ Heat on M1, Finish time of job on M2, Waiting Time (WT) and dead line and dead line violation is computed using computer programming as per the formulas mentioned above. In the set of heats below every four heats are scheduled on one set of M1 and M2. Total 12 LD converters and 12 Argon Rinsing units are used and parelley processing the heats. The elapsed time for each set of machines processing calculated. The results are shown in Table II. The average elapsed time is 112 minutes and it is shown in Table III.

TABLE II. The FCFS scheduling computation results

\begin{tabular}{|c|c|c|c|c|c|c|c|}
\hline $\begin{array}{c}\text { Job } \\
\text { ID }\end{array}$ & M1 & M2 & $\begin{array}{c}\text { Start Time } \\
\text { Of job on M1 }\end{array}$ & $\begin{array}{c}\text { Finish Time of } \\
\text { Job on M2 }\end{array}$ & WT & Dead Line & $\begin{array}{c}\text { Dead Line } \\
\text { Violation }\end{array}$ \\
\hline 0 & 15 & 32 & 0 & 47 & 0 & 94 & 0 \\
\hline 1 & 19 & 25 & 15 & 72 & 28 & 176 & 0 \\
\hline 2 & 17 & 25 & 34 & 97 & 55 & 168 & 0 \\
\hline 3 & 16 & 20 & 51 & 117 & 81 & 108 & 9 \\
\hline 4 & 17 & 20 & 0 & 37 & 0 & 74 & 0 \\
\hline
\end{tabular}




\begin{tabular}{|c|c|c|c|c|c|c|c|}
\hline 5 & 18 & 27 & 17 & 64 & 19 & 90 & 0 \\
\hline 6 & 18 & 12 & 35 & 76 & 46 & 90 & 0 \\
\hline 7 & 20 & 32 & 53 & 108 & 56 & 156 & 0 \\
\hline 8 & 19 & 17 & 0 & 36 & 0 & 144 & 0 \\
\hline 9 & 18 & 42 & 19 & 79 & 19 & 180 & 0 \\
\hline 10 & 17 & 14 & 37 & 93 & 62 & 62 & 31 \\
\hline 11 & 19 & 10 & 54 & 103 & 74 & 58 & 45 \\
\hline 12 & 15 & 17 & 0 & 32 & 0 & 96 & 0 \\
\hline 13 & 18 & 22 & 15 & 55 & 15 & 160 & 0 \\
\hline 14 & 17 & 18 & 33 & 73 & 38 & 70 & 3 \\
\hline 15 & 16 & 28 & 50 & 101 & 57 & 88 & 13 \\
\hline 16 & 16 & 25 & 0 & 41 & 0 & 123 & 0 \\
\hline 17 & 15 & 12 & 16 & 53 & 26 & 54 & 0 \\
\hline 18 & 17 & 22 & 31 & 75 & 36 & 156 & 0 \\
\hline 19 & 18 & 24 & 48 & 99 & 57 & 126 & 0 \\
\hline 20 & 15 & 14 & 0 & 29 & 0 & 87 & 0 \\
\hline 21 & 18 & 32 & 15 & 65 & 15 & 200 & 0 \\
\hline 22 & 17 & 95 & 33 & 160 & 48 & 224 & 0 \\
\hline 23 & 17 & 19 & 50 & 179 & 143 & 72 & 107 \\
\hline 24 & 23 & 19 & 0 & 42 & 0 & 126 & 0 \\
\hline 25 & 18 & 25 & 23 & 67 & 24 & 129 & 0 \\
\hline 26 & 10 & 19 & 41 & 86 & 57 & 87 & 0 \\
\hline 27 & 17 & 23 & 51 & 109 & 69 & 160 & 0 \\
\hline 28 & 17 & 14 & 0 & 31 & 0 & 93 & 0 \\
\hline 29 & 18 & 22 & 17 & 57 & 17 & 80 & 0 \\
\hline 30 & 22 & 14 & 35 & 71 & 35 & 108 & 0 \\
\hline 31 & 17 & 35 & 57 & 109 & 57 & 104 & 5 \\
\hline 32 & 17 & 22 & 0 & 39 & 0 & 78 & 0 \\
\hline 33 & 27 & 15 & 17 & 59 & 17 & 84 & 0 \\
\hline 34 & 18 & 8 & 44 & 70 & 44 & 78 & 0 \\
\hline 35 & 17 & 10 & 62 & 89 & 62 & 54 & 35 \\
\hline 36 & 18 & 9 & 0 & 27 & 0 & 108 & 0 \\
\hline 37 & 18 & 23 & 18 & 59 & 18 & 164 & 0 \\
\hline 38 & 17 & 11 & 36 & 70 & 42 & 84 & 0 \\
\hline 39 & 20 & 12 & 53 & 85 & 53 & 96 & 0 \\
\hline 40 & 21 & 25 & 0 & 46 & 0 & 184 & 0 \\
\hline 41 & 17 & 14 & 21 & 60 & 29 & 93 & 0 \\
\hline 42 & 18 & 32 & 38 & 92 & 42 & 150 & 0 \\
\hline 43 & 18 & 10 & 56 & 102 & 74 & 56 & 46 \\
\hline 44 & 18 & 13 & 0 & 31 & 0 & 62 & 0 \\
\hline 45 & 17 & 20 & 18 & 55 & 18 & 148 & 0 \\
\hline 46 & 17 & 20 & 35 & 75 & 38 & 74 & 1 \\
\hline \multirow[t]{2}{*}{47} & 10 & 25 & 52 & 100 & 65 & 105 & 0 \\
\hline & & & & & & & 295 \\
\hline
\end{tabular}


TABLE III. The sub scheduling sequences and elapsed time of schedule

\begin{tabular}{|l|l|}
\hline Sub Scheduling Sequences & Elapsed Time of Schedule \\
\hline FCFS Seq0 is $:\{0,1,2,3\}$ & 117 \\
\hline FCFS Seq1 is : $\{4,5,6,7\}$ & 108 \\
\hline FCFS Seq2 is : $\{8,9,10,11\}$ & 103 \\
\hline FCFS Seq3 is : $\{12,13,14,15\}$ & 101 \\
\hline FCFS Seq4 is : $\{16,17,18,19\}$ & 179 \\
\hline FCFS Seq5 is $:\{20,21,22,23\}$ & 109 \\
\hline FCFS Seq6 is : $\{24,25,26,27\}$ & 109 \\
\hline FCFS Seq8 is : $\{32,33,34,35\}$ & 89 \\
\hline FCFS Seq9 is : $\{36,37,38,39\}$ & 85 \\
\hline FCFS Seq10 is : $\{40,41,42,43\}$ & 102 \\
\hline FCFS Seq11 is : $\{44,45,46,47\}$ & 100 \\
\hline \multicolumn{1}{c}{ Average } & 112 \\
\hline
\end{tabular}

B. SJF Scheduling Results:

In this jobs/ heats are arranged in the order of Shortest Job i.e. total time of processing on LD Converters (M1) and Argon Rinsing Units (M2) together. After arranging the heats in Shortest Job First basis, each set of four heats are processed on one set of M1 and M2. The Start Time, Finish Time, Waiting Time, dead line and dead line violation is computed for each heat and shown in Table IV. The total averaged elapsed time is 109.97 minutes and it is shown in Table V.

TABLE IV. The results of SJF Scheduling

\begin{tabular}{|c|c|c|c|c|c|c|c|}
\hline $\begin{array}{c}\text { Job } \\
\text { ID }\end{array}$ & M1 & M2 & $\begin{array}{c}\text { Start Time } \\
\text { Of job on } \\
\text { M1 }\end{array}$ & $\begin{array}{c}\text { Finish Time of } \\
\text { Job on M2 }\end{array}$ & WT & $\begin{array}{c}\text { Dead } \\
\text { Line }\end{array}$ & $\begin{array}{c}\text { Dead Line } \\
\text { Violation }\end{array}$ \\
\hline 0 & 15 & 32 & 52 & 106 & 59 & 94 & 12 \\
\hline 1 & 19 & 25 & 52 & 99 & 55 & 176 & 0 \\
\hline 2 & 17 & 25 & 34 & 79 & 37 & 168 & 0 \\
\hline 3 & 16 & 20 & 10 & 49 & 13 & 108 & 0 \\
\hline 4 & 17 & 20 & 35 & 72 & 35 & 74 & 0 \\
\hline 5 & 18 & 27 & 47 & 97 & 52 & 90 & 7 \\
\hline 6 & 18 & 12 & 0 & 30 & 0 & 90 & 0 \\
\hline 7 & 20 & 32 & 53 & 110 & 58 & 156 & 0 \\
\hline 8 & 19 & 17 & 15 & 51 & 15 & 144 & 0 \\
\hline 9 & 18 & 42 & 57 & 118 & 58 & 180 & 0 \\
\hline 10 & 17 & 14 & 0 & 31 & 0 & 62 & 0 \\
\hline 11 & 19 & 10 & 0 & 29 & 0 & 58 & 0 \\
\hline 12 & 15 & 17 & 15 & 47 & 15 & 96 & 0 \\
\hline 13 & 18 & 22 & 28 & 75 & 35 & 160 & 0 \\
\hline 14 & 17 & 18 & 17 & 52 & 17 & 70 & 0 \\
\hline 15 & 16 & 28 & 55 & 105 & 61 & 88 & 17 \\
\hline 16 & 16 & 25 & 34 & 76 & 35 & 123 & 0 \\
\hline 17 & 15 & 12 & 0 & 27 & 0 & 54 & 0 \\
\hline 18 & 17 & 22 & 38 & 77 & 38 & 156 & 0 \\
\hline 19 & 18 & 24 & 35 & 78 & 36 & 126 & 0 \\
\hline 20 & 15 & 14 & 0 & 29 & 0 & 87 & 0 \\
\hline 21 & 18 & 32 & 44 & 104 & 54 & 200 & 0 \\
\hline & & & & & & & \\
\hline
\end{tabular}




\begin{tabular}{|l|c|c|c|c|c|c|c|}
\hline 22 & 17 & 95 & 51 & 174 & 62 & 224 & 0 \\
\hline 23 & 17 & 19 & 18 & 54 & 18 & 72 & 0 \\
\hline 24 & 23 & 19 & 34 & 76 & 34 & 126 & 0 \\
\hline 25 & 18 & 25 & 52 & 97 & 54 & 129 & 0 \\
\hline 26 & 10 & 19 & 0 & 29 & 0 & 87 & 0 \\
\hline 27 & 17 & 23 & 30 & 70 & 30 & 160 & 0 \\
\hline 28 & 17 & 14 & 0 & 31 & 0 & 93 & 0 \\
\hline 29 & 18 & 22 & 34 & 74 & 34 & 80 & 0 \\
\hline 30 & 22 & 14 & 19 & 55 & 19 & 108 & 0 \\
\hline 31 & 17 & 35 & 68 & 120 & 68 & 104 & 16 \\
\hline 32 & 17 & 22 & 35 & 74 & 35 & 78 & 0 \\
\hline 33 & 27 & 15 & 41 & 83 & 41 & 84 & 0 \\
\hline 34 & 18 & 8 & 0 & 26 & 0 & 78 & 0 \\
\hline 35 & 17 & 10 & 0 & 27 & 0 & 54 & 0 \\
\hline 36 & 18 & 9 & 0 & 27 & 0 & 108 & 0 \\
\hline 37 & 18 & 23 & 26 & 72 & 31 & 164 & 0 \\
\hline 38 & 17 & 11 & 0 & 28 & 0 & 84 & 0 \\
\hline 39 & 20 & 12 & 18 & 50 & 18 & 96 & 0 \\
\hline 40 & 21 & 25 & 46 & 100 & 54 & 184 & 0 \\
\hline 41 & 17 & 14 & 18 & 49 & 18 & 93 & 0 \\
\hline 42 & 18 & 32 & 50 & 108 & 58 & 150 & 0 \\
\hline 43 & 18 & 10 & 0 & 28 & 0 & 56 & 0 \\
\hline 44 & 18 & 13 & 17 & 48 & 17 & 62 & 0 \\
\hline 45 & 17 & 20 & 17 & 54 & 17 & 148 & 0 \\
\hline 46 & 17 & 20 & 17 & 54 & 17 & 74 & 0 \\
\hline 47 & 10 & 25 & 18 & 53 & 18 & 105 & 0 \\
\hline & & & & & & & $\mathbf{5 2}$ \\
\hline & & & & & & & \\
\hline
\end{tabular}

TABLE V. The sub scheduling sequence and elapsed time of schedule

\begin{tabular}{|l|l|}
\hline Sub Scheduling Sequences & Elapsed Time of Schedule \\
\hline SJF Seq0 is $:\{34,41,4,25\}$ & 97 \\
\hline SJF Seq1 is $:\{35,44,32,1\}$ & 99 \\
\hline SJF Seq2 is $:\{36,39,18,15\}$ & 105 \\
\hline SJF Seq3 is $:\{17,12,27,5\}$ & 97 \\
\hline SJF Seq4 is $:\{43,47,13,40\}$ & 100 \\
\hline SJF Seq5 is $:\{38,14,29,0\}$ & 106 \\
\hline SJF Seq6 is $:\{20,8,16,42\}$ & 108 \\
\hline SJF Seq7 is $:\{26,3,37,21\}$ & 104 \\
\hline SJF Seq8 is $:\{11,30,33,31\}$ & 120 \\
\hline SJF Seq9 is $:\{6,23,19,7\}$ & 110 \\
\hline SJF Seq10 is $:\{28,45,24,9\}$ & 118 \\
\hline SJF Seq1 1 is $:\{10,46,2,22\}$ & 174 \\
\hline \multicolumn{1}{|c|}{ Average } & 109.72 \\
\hline
\end{tabular}




\section{EDF Scheduling Results:}

The model randomly determines a factor for each job/heat and finds out a deadline time for each job/heat. After deadlines are arrived for each heat / job the jobs are ordered based on the deadline function in ascending order is sequenced. The scheduling model considers for each 4 jobs/heats it sequences on one stream of LD converter (M1) and Argon rinsing station (M2). The start time of Job on M1,Finish Time of Job on M2, Wait Time, Dead Line and Dead Line Violation is computed for each Job ID / Heat Id and tabulated in Table VI. The Dead Line Time (DLT) is computed with (M1 + M2) * Alpha and Alpha is a random value between 2 to 4. The Dead Line Function is computed based on the formula $\beta *$ DLT $+\gamma *(\mathrm{M} 1+\mathrm{M} 2)$. Where Beta and Gamma are chosen randomly such that Beta $+\mathrm{Gamma}=1$.In this research $\beta$ is taken as 0.4 and $\gamma$ is taken as 0.6 . Based on the Dead Line Function the sequence is ordered in ascending form. The dead line variation is difference between dead line and finish time of job on M2. If it is negative the dead line violation is zero otherwise it is same positive value. This indicates that the job/heat is completed within the dead line time then there is dead line violation. The average elapsed time is computed for the overall heats produced on all the set of machines and the value is 106 minutes, shown in Table VII.

TABLE VI. The Shortest Job Scheduling Results

\begin{tabular}{|c|c|c|c|c|c|c|c|c|}
\hline $\begin{array}{c}\text { Job } \\
\text { ID }\end{array}$ & M1 & M2 & $\begin{array}{c}\text { Start } \\
\text { Time } \\
\text { Of Mob } \\
\text { on }\end{array}$ & $\begin{array}{c}\text { Finish } \\
\text { Time of } \\
\text { Job on } \\
\text { M2 }\end{array}$ & WT & $\begin{array}{c}\text { Dead } \\
\text { Line( } \\
\text { (M12)* }\end{array}$ & $\begin{array}{c}\text { Alpha } \\
\text { (2 to }\end{array}$ & $\begin{array}{c}\text { 4) } \\
\text { Dead } \\
\text { Line } \\
\text { Violation }\end{array}$ \\
\hline 0 & 15 & 32 & 35 & 89 & 42 & 94 & 2 & 0 \\
\hline 1 & 19 & 25 & 67 & 111 & 67 & 176 & 4 & 0 \\
\hline 2 & 17 & 25 & 51 & 101 & 59 & 168 & 4 & 0 \\
\hline 3 & 16 & 20 & 37 & 73 & 37 & 108 & 3 & 0 \\
\hline 4 & 17 & 20 & 0 & 37 & 0 & 74 & 2 & 0 \\
\hline 5 & 18 & 27 & 17 & 62 & 17 & 90 & 2 & 0 \\
\hline 6 & 18 & 12 & 19 & 49 & 19 & 90 & 3 & 0 \\
\hline 7 & 20 & 32 & 52 & 119 & 67 & 156 & 3 & 0 \\
\hline 8 & 19 & 17 & 33 & 82 & 46 & 144 & 4 & 0 \\
\hline 9 & 18 & 42 & 50 & 121 & 61 & 180 & 3 & 0 \\
\hline 10 & 17 & 14 & 0 & 31 & 0 & 62 & 2 & 0 \\
\hline 11 & 19 & 10 & 0 & 29 & 0 & 58 & 2 & 0 \\
\hline 12 & 15 & 17 & 17 & 54 & 22 & 96 & 3 & 0 \\
\hline 13 & 18 & 22 & 50 & 111 & 71 & 160 & 4 & 0 \\
\hline 14 & 17 & 18 & 0 & 35 & 0 & 70 & 2 & 0 \\
\hline 15 & 16 & 28 & 17 & 65 & 21 & 88 & 2 & 0 \\
\hline 16 & 16 & 25 & 35 & 76 & 35 & 123 & 3 & 0 \\
\hline 17 & 15 & 12 & 0 & 27 & 0 & 54 & 2 & 0 \\
\hline 18 & 17 & 22 & 43 & 82 & 43 & 156 & 4 & 0 \\
\hline 19 & 18 & 24 & 37 & 79 & 37 & 126 & 3 & 0 \\
\hline 20 & 15 & 14 & 17 & 46 & 17 & 87 & 3 & 0 \\
\hline 21 & 18 & 32 & 52 & 114 & 64 & 200 & 4 & 0 \\
\hline 22 & 17 & 95 & 52 & 177 & 65 & 224 & 2 & 0 \\
\hline 23 & 17 & 19 & 0 & 36 & 0 & 72 & 2 & 0 \\
\hline 24 & 23 & 19 & 44 & 86 & 44 & 126 & 3 & 0 \\
\hline 25 & 18 & 25 & 32 & 79 & 36 & 129 & 3 & 0 \\
\hline 26 & 10 & 19 & 15 & 46 & 17 & 87 & 3 & 0 \\
\hline 27 & 17 & 23 & 53 & 96 & 56 & 160 & 4 & 0 \\
\hline 28 & 17 & 14 & 18 & 49 & 18 & 93 & 3 & 0 \\
\hline 29 & 18 & 22 & 17 & 57 & 17 & 80 & 2 & 0 \\
\hline
\end{tabular}




\begin{tabular}{|l|c|c|c|c|c|c|c|c|}
\hline 30 & 22 & 14 & 35 & 71 & 35 & 108 & 3 & 0 \\
\hline 31 & 17 & 35 & 35 & 87 & 35 & 104 & 2 & 0 \\
\hline 32 & 17 & 22 & 18 & 57 & 18 & 78 & 2 & 0 \\
\hline 33 & 27 & 15 & 17 & 59 & 17 & 84 & 2 & 0 \\
\hline 34 & 18 & 8 & 0 & 26 & 0 & 78 & 3 & 0 \\
\hline 35 & 17 & 10 & 0 & 27 & 0 & 54 & 2 & 0 \\
\hline 36 & 18 & 9 & 25 & 55 & 28 & 108 & 4 & 0 \\
\hline 37 & 18 & 23 & 57 & 98 & 57 & 164 & 4 & 0 \\
\hline 38 & 17 & 11 & 0 & 28 & 0 & 84 & 3 & 0 \\
\hline 39 & 20 & 12 & 17 & 49 & 17 & 96 & 3 & 0 \\
\hline 40 & 21 & 25 & 55 & 104 & 58 & 184 & 4 & 0 \\
\hline 41 & 17 & 14 & 18 & 49 & 18 & 93 & 3 & 0 \\
\hline 42 & 18 & 32 & 42 & 103 & 53 & 150 & 3 & 0 \\
\hline 43 & 18 & 10 & 0 & 28 & 0 & 56 & 2 & 0 \\
\hline 44 & 18 & 13 & 0 & 31 & 0 & 62 & 2 & 0 \\
\hline 45 & 17 & 20 & 35 & 82 & 45 & 148 & 4 & 0 \\
\hline 46 & 17 & 20 & 0 & 37 & 0 & 74 & 2 & 0 \\
\hline 47 & 10 & 25 & 32 & 71 & 36 & 105 & 3 & 0 \\
\hline & & & & & & & & 0 \\
\hline
\end{tabular}

TABLE VII. The sub scheduling Sequence and Elapsed Time of Schedule

\begin{tabular}{|l|l|}
\hline \multicolumn{1}{|c|}{ Sub Scheduling Sequences } & Elapsed Time of Schedule \\
\hline EDF Seq0 is $:\{17,26,36,18\}$ & 82 \\
\hline EDF Seq1 is $:\{35,20,47,42\}$ & 103 \\
\hline EDF Seq2 is $:\{43,32,0,13\}$ & 111 \\
\hline EDF Seq3 is $:\{11,6,3,27\}$ & 96 \\
\hline EDF Seq4 is $:\{10,29,30,37\}$ & 98 \\
\hline EDF Seq5 is $:\{44,28,31,7\}$ & 119 \\
\hline EDF Seq6 is $:\{34,41,16,2\}$ & 101 \\
\hline EDF Seq7 is $:\{14,33,24,1\}$ & 111 \\
\hline EDF Seq8 is $:\{23,39,19,40\}$ & 104 \\
\hline EDF Seq9 is $:\{4,12,25,9\}$ & 121 \\
\hline EDF Seq10 is $:\{46,15,8,21\}$ & 114 \\
\hline EDF Seq11 is $:\{38,5,45,22\}$ & 117 \\
\hline \multicolumn{1}{|c|}{ Average } & 106 \\
\hline
\end{tabular}

\section{Comparison of Results}

The Average Elapsed Time, Total Dead Line Violation ofthe EDF scheduling model when compared with FCFS and SJF is shown in Table VIII. The graphical comparison of Scheduling Model and Average Elapsed Time is shown in Figure 2. The graphical Comparison of Scheduling moel and Total Dead line Violation is shown in Figure 3. These values indicates that EDF (106) scheduling model is better than the FCFS (109) and SJF (112) . The total deadline violation values indicates that the EDF (0.0) scheduling is giving much better results when compared with SJF (52.0) and FCFS (295.0). 
TABLE VIII. The scheduling model viz. Average Elapsed Time, Total Dead Line Violation

\begin{tabular}{|l|l|l|}
\hline Scheduling Model & Average Elapsed Time & Total Deadline Violation \\
\hline FCFS & 109.00 & 295.00 \\
\hline SJF & 112.00 & 52.00 \\
\hline EDF & 106.00 & 0.00 \\
\hline
\end{tabular}

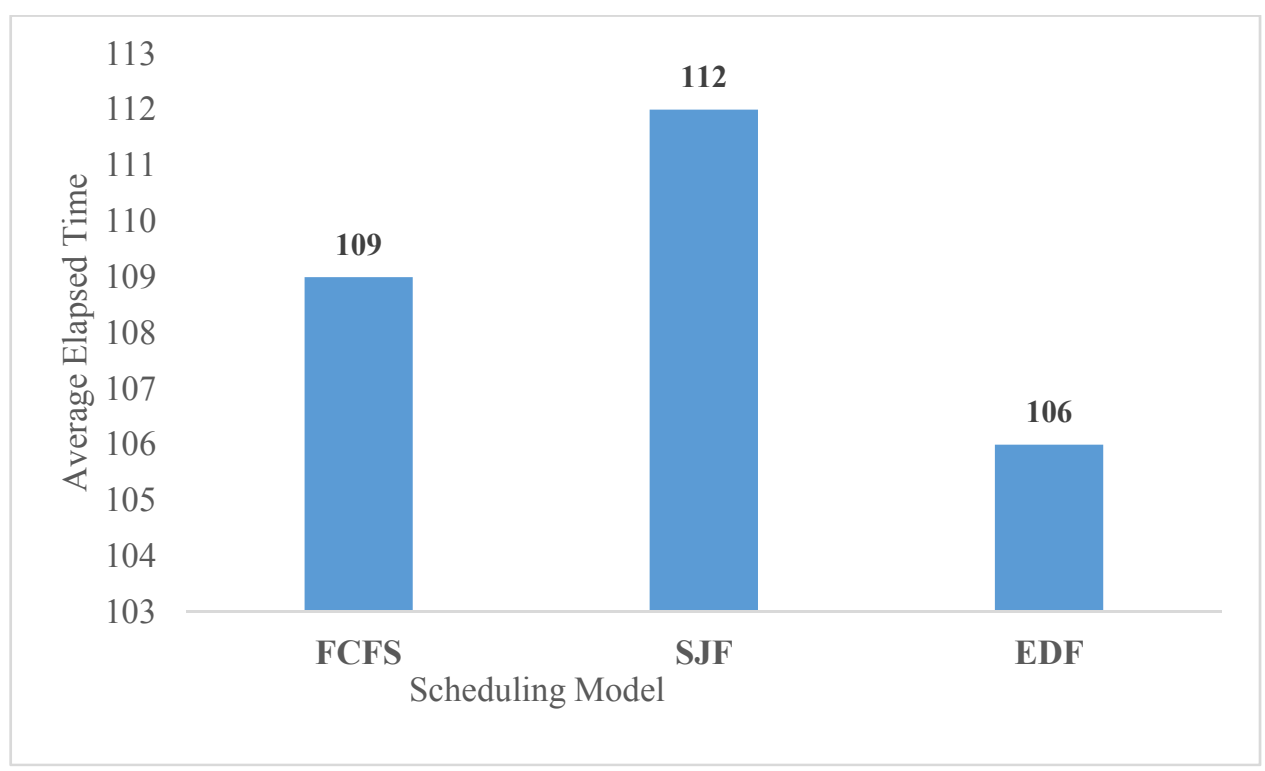

Fig.2 Averaged Elapsed Time variation with Scheduling Model

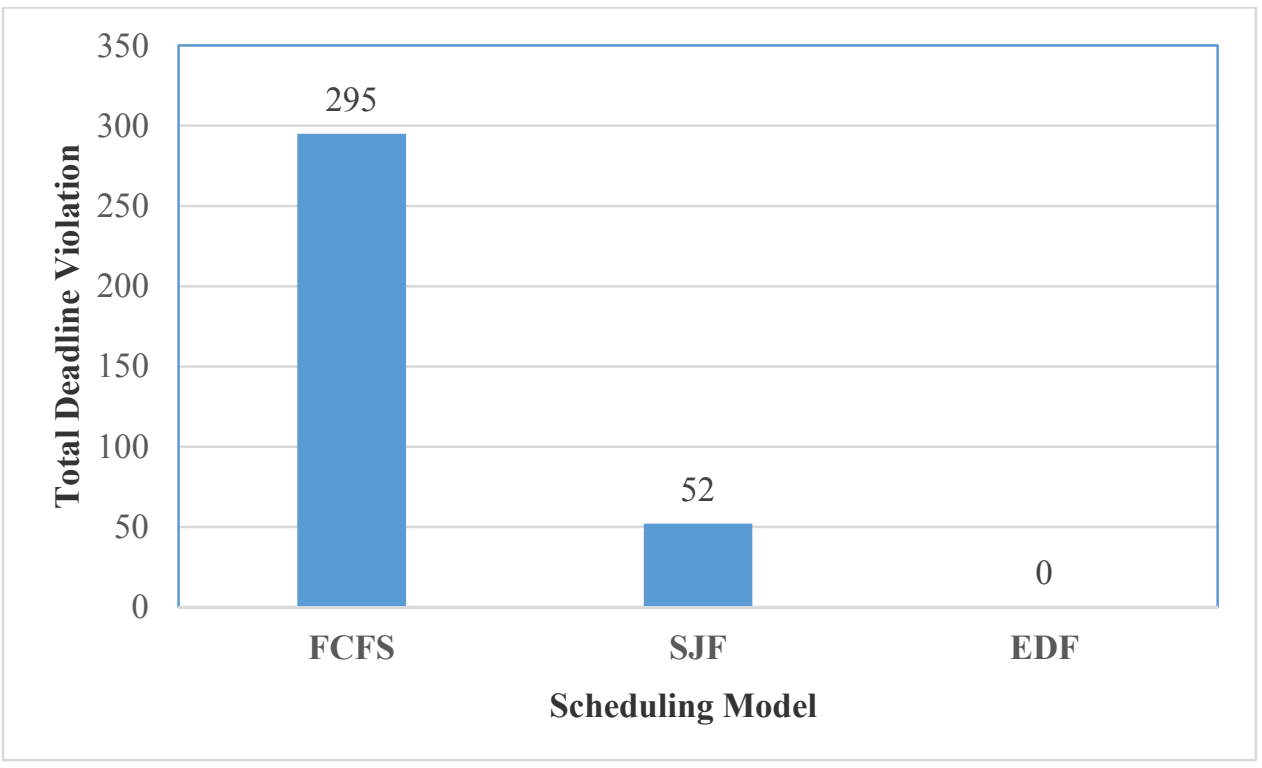

Fig.3 Variation of Total Deadline variation with scheduling model

\section{CONCLUSION}

In this research EDF model is used for scheduling the heats at LD converter and ARS Stations. The scheduling results are compared with other conventional models viz FCFS and SJF. The result shows that EDF Model performance in terms of total deadline violation is much better than other FCFS and SJF, this implies that the deviation from deadline time is much less and better than others. The average elapsed time for a job in the case of EDF is better than FCFS and SJF. 


\section{REFERENCES}

[1] H. B. Wang, A. J. Xu, D. F. He, "Solving Steel-Making and Continuous-Casting Scheduling Problem with Fuzzy Processing and Delivery Time Using Genetic Algorithm", Advanced Materials Research, Vols. 139-141, PP: 1679-1683, 2010

[2] Worapradya.K, Thanakikasem P etal, "Optimizing Steel Production Schedules via a hierarchical genetic algorithm", South African Journal of Industrial Engineering, Vol :25, PP: 209-221, 2014

[3] Iiro Harjunkoski, Guido Sand , "Flexible and configurable MILP-Models for Meltshop Scheduling Optimization", 18th European Symposium on Computer Aided Process Engineering, 2008

[4] Vikas Kumar, Shashi Kumar,M.K.Tiwari \& F.T.S Chan, " Auction-based approach to resolve the scheduling problem in the steel making process", International Journal of Production Research, Vol: 44:8,PP:1503-1522, 2006

[5] Kiatkajohn Worapradya, Purit Thanakijkasem, "Proactive scheduling for Steelmaking-continuous Casting Plant with Uncertain Machine Breakdown Using Distribution-Based Robustness and Decomposed Artificial Neural Network", Asia Pacific Journal of Operational Research, Vol:32, 2015

[6] PA Guegler and FJ Vasko, "Metaheuristics for melt shop scheduling in the steel Industry", Journal of the Operational Research Society, Vol:58, PP:791-796, June 2007

[7] Hubert Missbauer, Wolfgang Hauber \& Werner Stadler, "A scheduling system for the steel making continuous casting process. A case study from the steel-making industry", International Journal of Production Research, 47:15,PP:4147-4172, 2009

[8] Arezoo Atighehchian, Mehdi Bijari, Hamed Tarkesh , "Novel Hybrid Algorithm for Scheduling Steel-Making Continuous Casting Production",Computers and Operations research, Vol: 36:8,PP:2450-2461, 2009

[9] Kebin Lu, Kewei Huang and Dingwei Wang, "Integrated Scheduling System on Steel-Making and Continuous Casting with Application", Proceedings of the 17th World Congress the International federation of automatic control, Vol:17,PP:9149-9150, 2008

[10] L.Sun,T.Chai,P.B.Luh, "Scheduling of Steel-making and continuous casting system using surrogate sub-gradient algorithm for lagrangian relaxation", Automation Science and Engineering IEEE conference,PP: 885-890, 2010

[11] Liangiang Sun, Wei Liu,B.Xu, Tianyou Chai, "The scheduling of steel making and continuous casting process using branch and cut method via CPLEX optimization", 5th International Conference on Computers Sciences and Convergence Information Technology, PP:716-721, 2010

[12] Xiu-ying wang etal, "Intelligent optimization scheduling of steelmaking and continuous casting production and its application", 8th World congress on Intelligent Control and Automation, PP: 288-294, 2010

[13] Dario Pacciarelli, Marco Pranzo, "Production Scheduling in a steel Making - continuous casting plant", Journal of Computers \&Chemical Engineering, Vo: 28:12, PP: 2823-2835, 2014

[14] IeLi,Xin Xiao,Qiuhua Tang and Christodoulos A.Floudas, " Production Scheduling of a Large Scale Steel Makin Continuous Casting Process via Unit-Specific Event-Based Continuous -Time Models: Short Term and Medium-Term Scheduling", Industrial \& Engineering Chemistry Research, 51:21, PP: 7300-7319, 2012

[15] X.F.Li, L.Y.Xu,H.H.Shao, D.X.Ren,"Dynamic model of steel-making and continuous casting system and hierarchic algorithm of dynamic scheduling", Journal of Shanghai Hiaotong University, 35:11, PP: 1658-1662, 2001

\section{AUTHOR PROFILE}

P.Chandra sekhar : He has obtained his B.Tech(Metallurgy) from NIT, Warangal, M.Tech(Metallurgy) from IIT , Bombay, Post Graduatediploma in Computer Science from Central University, Hyderabad. He has 28 years of industrial experience working in Visakhapatnam Steel Plant, Visakhapatnam, Andhra Pradesh, India in various department like Quality Assurance and Technology Department, IT. He is presently working as Dy.General Manager (IT).

Prof.P.SureshVarma : M.Tech(CST),Ph.D.(CSE),FIE,FIETE,SMCSI,AMIE,MISTE,SMORSI,MIISA,MISCA Professor of Computer Science and Engineering, Principal, University College of Engineering, \& Dean Faculty of Engineering and Technology \& Webmaster of University Website,Adikaviannaya University ,Rajahmundry,AP,India 